\title{
CHARACTERIZING A CIRCLE WITH THE DOUBLE MIDSET PROPERTY
}

\author{
L. D. LOVELAND AND J. E. VALENTINE
}

ABSTRACT. A short and elementary proof is given to show that a space $X$ is a circle with the natural geodesic metric if $X$ is a nondegenerate, complete, convex metric space with the double midset property.

In 1970 Berard [1] announced that a complete convex metric space having the double midset property must be a topological simple closed curve. Based on a manuscript [2] subsequently received from him, Loveland and Valentine [5] showed that under Berard's hypothesis the space is actually isometric to a circle having the natural geodesic metric. Although Berard's manuscript was not published, a paper by Berard and Nitka [3] has recently appeared in which the isometry is established. However a short and elementary proof can be obtained by quoting Theorem 1 of [6] and Theorem 2 of [5], with some extra work. Rather than proceed in this manner we have endeavored to make this note largely self-contained. Thus we adapt some proofs to our situation and give them here.

The midset (called the "bisector" in [3]) of two points $a$ and $b$ of a metric space $X$ is the set of all $x$ in $X$ such that the distances $a x$ and $b x$ are equal, and $X$ is said to have the double midset property (DMP) if, for every pair of distinct points $a$ and $b$ of $X$, the midset $M(a, b)$ of $a$ and $b$ consists of two points.

In the remainder of the paper, $X$ will denote a nondegenerate, complete, convex, metric space having the DMP. It is easy to see that the "complete, convex" hypothesis can be replaced by "segment-convex" as is done in [3]. The essential hypothesis is that $X$ contain with two of its points a segment joining them (see [4, Theorem 14.1, p. 41]).

Lemma 1. The space $X$ contains a simple closed curve.

Proof. Let $a$ and $b$ be points of $X, S$ a segment with endpoints $a$ and $b$, and $M(a, b)=\left\{m_{1}, m_{2}\right\}$. Since $S$ cannot have two midpoints, $m_{2} \notin S$. Let $S^{\prime}$ be the union of two segments $S_{1}$ and $S_{2}$ having endpoints $\left\{a, m_{2}\right\}$ and $\left\{b, m_{2}\right\}$, respectively. Obviously $S \cup S^{\prime}$ contains a simple closed curve unless $S_{1}$ and $S_{2}$ share a segment $S_{3}$ with endpoints $m_{1}$ and $m_{2}$. However

Received by the editors October 6, 1974.

AMS (MOS) subject classifications (1970). Prinary 52A05, 54E40.

Key words and phrases. Convex, midsets, bisectors, simple closed curves, double midset property. 
$S_{3} \subset S_{1} \cap S_{2}$ implies $S_{3} \subset M(a, b)$, contradicting the DMP.

Lemma 2 [6, Theorem 1]. The space $X$ is a topological simple closed curve.

Proof. From Lemma 1, $X$ contains a simple closed curve J. Suppose there is a point $x$ in $X-J$. There cannot exist two points $a$ and $b$ of $J$ equidistant from $x$, for then $M(a, b)$ would contain $x$ and could intersect $J$ at most once. This would contradict the fact that $M(a, b)$ separates a from $b$ in $X$. Thus the function $g: J \rightarrow R$, defined by $g(t)=x t$, is a continuous injection of $J$ into the real line. This is impossible since $g$ is a homeomorphism under these conditions.

Main theorem [5, Theorem 2]. The space $X$ is isometric to a circle having the natural geodesic metric.

Proof. Let $a$ and $x$ be two points of the simple closed curve $X$ (see Lemma 2), and let $S(a, b)$ be a maximal (with respect to inclusion) segment containing $x$ and having $a$ as an endpoint. Let $\left\{x_{i}\right\}$ be a monotone sequence of points of the open arc $X-S(a, b)$ converging to $b$. Since $S(a, b) \cap$ $S\left(a, x_{n}\right)=\{a\}$, we see that the closure of $\bigcup_{i=1}^{\infty} S\left(a, x_{i}\right)$ is a segment $S^{\prime}(a, b)$ and that $X=S(a, b) \cup S^{\prime}(a, b)$. Let $C$ be a circle in $E^{2}$ of radius $a b / \pi$ with the geodesic metric. Let $f$ be a homeomorphism taking $X$ onto $C$ such that $f \mid S(a, b)$ and $f \mid S^{\prime}(a, b)$ are both isometries onto semicircles of $C$. To show that $f$ is an isometry it suffices to check that $f(x) f(y)=x y$ whenever $x$ and $y$ are chosen in the interiors of $S(a, b)$ and $S^{\prime}(a, b)$, respectively. We denote $f(z)$ by $z^{\prime}$, and we define $p q r$ to mean $p q+q r=p r$. We may assume that xay holds. If $x^{\prime} a^{\prime} y^{\prime}$ also holds, then $x y=x^{\prime} y^{\prime}$ as desired. Otherwise we have $x^{\prime} b^{\prime} y^{\prime}$, and we now show this implies $x b y$ from which $x y=$ $x^{\prime} y^{\prime}$. Suppose $x b+b y>x y$. Since $x y=x a+a y$, this leads to the contradiction that $x^{\prime} y^{\prime}>x^{\prime} a^{\prime}+a^{\prime} y^{\prime}$.

\section{REFERENCES}

1. A. D. Berard, Jr., Characterizations of metric spaces by the use of their midsets: One-spheres, Notices Amer. Math. Soc. 19 (1972), A-198. Abstract \#691-54-11.

2. Characterizations of metric spaces by the use of their midsets: One-spheres (Un published manuscript, 1-14).

3. A. D. Berard, Jr. and W. Nitka, A new definition of the circle by the use of bisectors, Fund. Math. 85 (1974), 49-55.

4. L. M. Blumenthal, Theory and applications of distance geometry, Clarendon Press, Oxford, 1953. MR 14, 1009.

5. L. D. Loveland and J. E. Valentine, Convex metric spaces with 0-dimensional midsets, Proc. Amer. Math. Soc. 37 (1973), 568-571. MR 46 \#9915.

6. L. D. Loveland and S. G. Wayment, Characterizing a curve with the double midset property, Amer. Math. Monthly 81 (1974), 1003-1006. 